Supplemental Materials for:

\title{
The Palladium-Catalyzed Enantioselective Oxidation of Alcohols: A Dramatic Rate Acceleration by $\mathrm{Cs}_{2} \mathrm{CO}_{3} / t$-BuOH
}

\author{
Jeffrey T. Bagdanoff, Eric M. Ferreira and Brian M. Stoltz* \\ The Arnold and Mabel Laboratories of Chemical Synthesis, Division of Chemistry and Chemical \\ Engineering, California Institute of Technology, Pasadena, California, 91125, USA
}

Material and Methods. Unless stated otherwise, reactions were performed in oven-dried glassware, under an atmosphere of oxygen, using freshly distilled solvents. Although we have never experienced an accident, all reactions must be performed with appropriate caution in a fume hood due to the flammable nature of mixtures of oxygen and organic solvents. All other commercially obtained reagents were used as received. Reaction temperatures were controlled by an IKAmag temperature modulator. Thin-layer chromatography (TLC) was performed using E. Merck silica gel 60 F254 precoated plates $(0.25 \mathrm{~mm})$. ICN Silica gel (particle size 0.032-0.063 mm) was used for flash chromatography. Analytical chiral HPLC was performed on a Chiralcel OJ, AS, AD, OB-H or OD-H column (each is $4.6 \mathrm{~mm}$ x $25 \mathrm{~cm}$ ) obtained from Daicel Chemical Industries, Ltd. Analytical achiral GC was performed using an Agilent DB-WAX (30.0 m x $0.25 \mathrm{~m})$ column. Analytical chiral GC was carried out using a Chiraldex B-DM column (30.0 m x $0.25 \mathrm{~mm}$ ) purchased from Bodman Industries. Commercially available racemic alcohols in Table 3 (entries 1, 2, 3, 6, 7, 8, and 9) were purchased from the Sigma-Aldrich Chemical Company, Milwaukee, WI. Non-commercially available racemic alcohols used in Table 3 (corresponding to entries 4, 6, and 10) were prepared as previously described. ${ }^{1}$ Commercially available samples of enantiopure alcohols for analytical comparison purposes (entries 1, 7, 8, and 9) were purchased from the Sigma-Aldrich Chemical Company, Milwaukee, WI. Non-commercially available enantiopure alcohols prepared by palladium-catalyzed oxidative kinetic resolution (Table 3 entries $2,{ }^{2} 3,{ }^{3} 6,{ }^{4}$ and $10^{1,5}$ ) were compared by optical rotation to known values. The previously unknown enantiopure alcohols (Table 3, entries 4 and 5) were assigned absolute stereochemisty by analogy to assigned resolution products.

\footnotetext{
${ }^{1}$ (a) Ruble, J. C.; Latham, H. A.; Fu, G. C. J. Am. Chem. Soc. 1997, 119, 1492. (b) Ruble, J. C., Tweddell, J.; Fu, G. C. J. Org. Chem. 1998, 63, 2794.

${ }^{2}$ Nakamura, K.; Inoue, Y.; Matsuda, T.; Misawa, I. J. Chem. Soc., Perkin. Trans. 1 1999, 2397.

${ }^{3}$ Nieduzak, T. R.; Margolin, A. L. Tetrahedron: Asymmetry 1991, 2, 113.

${ }^{4}$ Nakamura, K.; Matsuda, T. J. Org. Chem. 1998, 63, 8957.

${ }^{5}$ Argus, C. L.; Cort, L. A.; Howard, T. J.; Loc, L. B. J. Chem. Soc. 1960, 1195.
} 


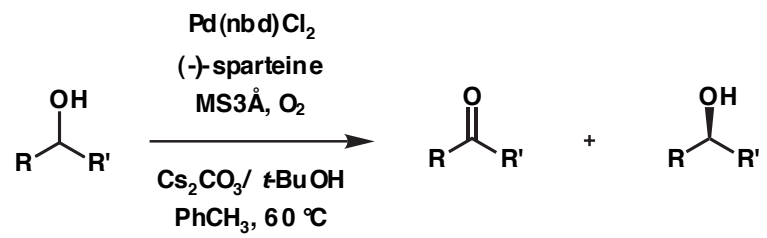

\section{General Procedure for the Rate Accelerated Oxidative Kinetic Resolution of Secondary Alcohols.}

An oven dried reaction tube (outer diameter $16 \mathrm{~mm}$, length $120 \mathrm{~mm}$ ) equipped with a magnetic stir bar was charged with oven dried powdered molecular sieves (MS3 $\AA$, $0.5 \mathrm{~g}$ ). After cooling, $\mathrm{Pd}(\mathrm{nbd}) \mathrm{Cl}_{2}$ complex ( $0.05 \mathrm{mmol}, 0.05$ equiv) was added followed by toluene $(2.0 \mathrm{~mL})$, and (-)-sparteine ( $0.2 \mathrm{mmol}, 0.20$ equiv). The flask was cooled to $-78^{\circ} \mathrm{C}$, then vacuum evacuated and filled with $\mathrm{O}_{2}(3 \mathrm{x}$, balloon), then heated to 60 ${ }^{\circ} \mathrm{C}$ for $15 \mathrm{~min}$. Powdered anhydrous $\mathrm{Cs}_{2} \mathrm{CO}_{3}\left(0.5 \mathrm{mmol}, 0.5\right.$ equiv.), ${ }^{6}$ a toluene soultion $(2.0 \mathrm{~mL})$ of the alcohol (1.0 mmol, 1.0 equiv), and $t-\mathrm{BuOH}(1.5 \mathrm{mmol}, 1.5$ equiv.) was introduced and the reaction mixture was maintained at $60{ }^{\circ} \mathrm{C}$. The reaction was monitored by standard analytical techniques (TLC, GC, ${ }^{1} \mathrm{H}-$ NMR, and HPLC) for \% conversion and enantiomeric excess values. Aliquots of the reaction mixture $(0.2$ $\mathrm{mL}$ ) were collected after $2 \mathrm{~h}, 4 \mathrm{~h}, 8 \mathrm{~h}, 12 \mathrm{~h}, 15 \mathrm{~h}$, and $18 \mathrm{~h}$ depending on the course of the reaction (typically three aliquots per run). Each aliquot was filtered through a small plug of silica gel ( $\mathrm{Et}_{2} \mathrm{O}$ eluent), evaporated and analyzed. ${ }^{7}$

Optical rotations for new compounds:
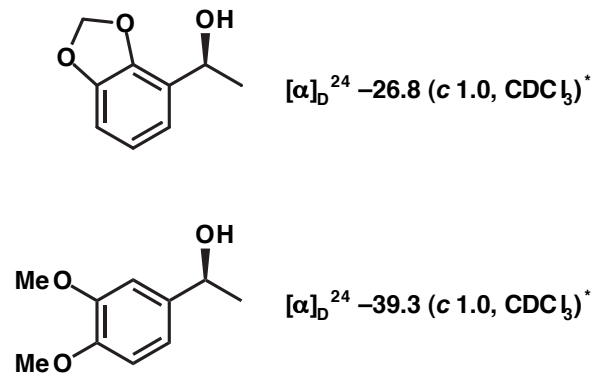

*absolute stereochemistry of these molecules assigned by analogy.

\footnotetext{
${ }^{6}$ For experiments that probed the effect of other addiives, the appropriate base/alcohol was used in the same general procedure.

${ }^{7}$ Percent conversions were measured by GC integration of the alcohol and the ketone peaks, correcting for response factors (for conditions see SM Table 2).
} 
Table SM 1. Methods utilized for the deter mi nation of enantiomeric excess.

\begin{tabular}{llcc}
\hline entry & ee Assay & Conditions & $\begin{array}{c}\text { Retention Time } \\
\text { of }(R) \text { isomer (min) }\end{array}$ \\
\hline of $(S)$ isomer (min)
\end{tabular}

3.<smiles>CC(O)c1ccc(F)cc1</smiles>

HPLC

Ch iraldex AS

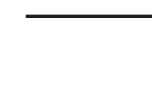

4.<smiles>COc1ccc(C(C)O)cc1OC</smiles>

HPL C ChiralcelOD-H 5\% EtOH/hexane 4. $\mathrm{MeO}$

5.<smiles>CC(O)c1cccc2c1OCO2</smiles>

HPLC Chir alceIOD-H 1\% EtOH/hexane $1.0 \mathrm{~m} \mathrm{~L} / \mathrm{min}$

6.<smiles>CC(O)c1ccc2ccccc2c1</smiles>

\begin{abstract}
HPLC
\end{abstract}
Chiralcel AS
2\% 2-prop anol/hexane

$1.0 \mathrm{~mL} / \mathrm{m} \mathrm{n}$
18.60

21.13
7.<smiles>CCC(O)c1ccccc1</smiles>

HPLC Chir alce IOB-H
1\% EtOH/hexane

$1.0 \mathrm{~m} \mathrm{L/min}$
25.31 
Table SM 1 (continued).

\begin{tabular}{|c|c|c|c|c|c|}
\hline en try & Substrate & ee Assay & Conditions & $\begin{array}{c}\text { Retention Time } \\
\text { of }(R) \text { is omer }(\mathrm{m} \text { in) }\end{array}$ & $\begin{array}{c}\text { Retention Time } \\
\text { of }(S) \text { is om er (min) }\end{array}$ \\
\hline 8. & & $\begin{array}{c}\text { HPLC } \\
\text { Chi ralcel AS }\end{array}$ & $\begin{array}{c}2 \% \text { 2-propanol/hexane } \\
1.0 \mathrm{~m} \mathrm{~L} / \mathrm{min}\end{array}$ & 15.84 & 20.10 \\
\hline 9. & & $\begin{array}{c}\text { HPLC } \\
\text { Chi ralcel AS }\end{array}$ & $\begin{array}{c}2 \% \mathrm{EtOH} / \mathrm{hexane} \\
1.0 \mathrm{~mL} / \mathrm{min}\end{array}$ & 12.21 & 16.48 \\
\hline 10. & & $\begin{array}{c}\text { HPLC } \\
\text { Chiralcel OD-H }\end{array}$ & $\begin{array}{c}2 \% \mathrm{EtOH} / \mathrm{hexane} \\
1.0 \mathrm{~mL} / \mathrm{min}\end{array}$ & 17.58 & 20.88 \\
\hline
\end{tabular}


Table SM 2. Metho ds utilized for the deter mination of $\%$ con version.

entry alcohol

3.<smiles>CC(O)c1ccc(F)cc1</smiles><smiles>CC(=O)c1ccc(F)cc1</smiles>

$70{ }^{\circ} \mathrm{C}, 15 \mathrm{~min}$; $7.0^{\circ} \mathrm{C} / \mathrm{min}$ to $220^{\circ} \mathrm{C}$

$1.0 \mathrm{~mL} / \mathrm{min}$ carrier gas flow
29.42

25.46
4.<smiles>COc1ccc(C(C)O)cc1OC</smiles><smiles>COc1ccc(C(C)=O)cc1OC</smiles><smiles>CC(=O)c1cccc2c1OCO2</smiles>

$70{ }^{\circ} \mathrm{C}, 0 \mathrm{~min}$ $3.0^{\circ} \mathrm{C} / \mathrm{min}$ to $270^{\circ} \mathrm{C}$

$1.0 \mathrm{~mL} / \mathrm{min}$ $3.0^{\circ} \mathrm{C} / \mathrm{min}$ to $270^{\circ} \mathrm{C}$

$1.0 \mathrm{~mL} / \mathrm{min}$ carrier gas flow

37.60
5.<smiles>CC(O)c1cccc2c1OCO2</smiles>

6.<smiles>CC(O)c1ccc2ccccc2c1</smiles><smiles>CC(=O)c1ccc2ccccc2c1</smiles>

$70{ }^{\circ} \mathrm{C}, 15 \mathrm{~min}$; $7.0^{\circ} \mathrm{C} / \mathrm{min}$ to $220^{\circ} \mathrm{C}$

42. 00

$1.0 \mathrm{~mL} / \mathrm{min}$ carrier gas flow

36.32

35.04 
Table SM 2 (continued).

entry

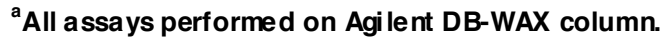

